\title{
AVALIAÇÃO DA RESIDÊNCIA MULTIPROFISSIONAL EM SAÚDE COLETIVA: UMA ANÁLISE PERCEPTIVA DOS PROFISSIONAIS ENVOLVIDOS NO PROGRAMA
}

\author{
ASSESSMENT OF MULTIPROFESSIONAL RESIDENCE IN \\ COLLECTIVE HEALTH: A PERCEPTIVE ANALYSIS OF THE \\ PROFESSIONALS INVOLVED IN THE PROGRAM
}

\author{
Sérgia Landara Bezerra Soares ${ }^{1}$ \\ Marleide Lopes Ferreira ${ }^{2}$ \\ Rodolfo de Abreu Carolino ${ }^{3}$ \\ Fernanda Alexandre Lima e Silva ${ }^{4}$ \\ Daniel de Mélo Carvalho ${ }^{5}$ \\ Anacácia Pessoa Leite ${ }^{6}$ \\ Lívia Pereira Brocos Pires ${ }^{7}$
}

RESUMO: Introdução: Os Programas de Residência Multiprofissional (PRM) em Saúde são mecanismos de cooperação intersetorial que visam a inserção de jovens profissionais da saúde qualificados para exercer suas profissões no mercado de trabalho, particularmente preparados para trabalhar e contribuir para o desenvolvimento e construção do SUS. O Programa de Residência em Saúde Coletiva com foco em gestão e planejamento em saúde permite projetar a habitual rotina reproduzida no dia a dia dos serviços de saúde, como antigas práticas da saúde pública tradicional para uma expectativa de inovação que modifique os modelos de cuidado e gestão em saúde. Objetivo: Avaliar a atuação, atividades, a

\footnotetext{
Graduada em Odontologia pela Universidade Federal de Campina Grande. landarasoares@gmail.com.

2 Graduada em Odontologia pela Faculdade Santa Maria de Cajazeiras. Marleidelopes1979@gmail.com.

${ }^{3}$ Mestre em Odontologia pela Universidade Estadual da Paraíba, docente do curso de Odontologia da Faculdade Santa Maria de Cajazeiras. rodolfoorg@yahoo.com.br.

4 Graduada em Medicina pelo Centro Universitário Cesmac - Maceió - AL. fernadalexandree@hotmail.com

5 Graduado em Medicina pelo Centro Universitário Cesmac - Maceió - AL. danielkrvalho@hotmail.com.

6 Graduada em Medicina pelo Centro Universitário Cesmac - Maceió - AL. anacacioleite@hotmail.com.

${ }^{7}$ Mestre em Odontologia pela Universidade Potiguar, docente do curso de Odontologia da Faculdade Santa Maria de Cajazeiras. liviabrocos@gmail.com.
} 
interação e estrutura organizacional de um PRM. Metodologia: O estudo transversal foi realizado no Alto Sertão da Paraíba com amostra $(n=29)$ participante do Programa de Residência Multiprofissional em Saúde Coletiva (tutores, residentes, preceptores e coordenadores). Esses profissionais responderam a um questionário autoaplicado que envolvia questões avaliativas no âmbito estrutural, organizacional e de atuação do PRM. A análise dos dados foi obtida a partir da frequência absoluta e relativa dos dados. Resultado: Os resultados mostraram dados quantitativos divididos em três diferentes dimensões do programa, a dimensão da gestão, da estrutura organizacional e do processo pedagógico. Quanto ao grau de envolvimento entre tutores, preceptores e IES na implantação do PRMSC, 50\% consideraram que houve um envolvimento parcial na implantação. No quesito vínculo entre a IES com a RMSC, a totalidade dos participantes respondeu que esse vínculo se faz presente. $\mathrm{Na}$ vertente da participação dos residentes, tutores e preceptores nas avaliações sistemáticas do PRMSC, 42,9\% consideraram que existe pouca participação. Sobre o processo de avaliação do PRMSC, 28,6\% dos profissionais envolvidos consideraram que as avaliações são previstas e realizadas ao longo do programa, entretanto uma parcela semelhante de profissionais considerou que essas avaliações são previstas e não realizadas. A partir desses dados foi possível a identificação e discussão de fragilidades que devem ser superadas para 0 fortalecimento do PRMSC. Conclusão: Conclui-se que os instrumentos avaliativos são importantes para a identificação de pontos que precisam ser melhorados dentro do programa, utilizando-se da percepção da amostra inserida no programa para direcionamentos que visem promover melhorias nos pontos de fragilidades encontrados, possibilitando assim o fortalecimento e desenvolvimento do programa.

Palavras-chave: Internato e Residência, Saúde Pública, Avaliação de programa.

ABSTRACT: Introduction: The Multiprofessional Residency Programs (MRP) in Health are mechanisms of intersectoral cooperation aimed at the insertion of young health professionals qualified to exercise their professions in the labor market, particularly prepared to work and contribute to the development and construction of the SUS. The Public Health Residency Program focused on health management and planning allows designing the usual routine reproduced in the day-to-day of health services, as old practices of traditional public health, for an expectation of innovation that modifies the models of health care and management. Objective: To evaluate the performance, activities, interaction and organizational structure of a PRM. Methodology: The cross-sectional study was conducted in the High Dryland of Paraiba with a sample $(n=29)$ participating in the Multiprofessional Residency Program in Public Health (tutors, residents, preceptors and coordinators). These professionals answered a self-administered questionnaire that involved evaluative questions in the structural, organizational and operational scope of the PRM. Data analysis was obtained from the absolute and relative frequency of the data. Result: The results showed quantitative data, divided into three different dimensions of the program, the dimension of management, organizational structure and pedagogical process. Regarding the degree of involvement between tutors, preceptors and HEls in the implementation of the PRMSC, 50\% considered that there was a partial involvement in the implementation. Regarding the link between the HEI and the 
RMSC, all participants responded that this link is present. Regarding the participation of residents, tutors and preceptors in the PRMSC's systematic evaluations, $42.9 \%$ considered that there is little participation. Regarding the PRMSC evaluation process, $28.6 \%$ of the professionals involved considered that the evaluations are planned and conducted throughout the program, however a similar portion of professionals considered that these evaluations are foreseen and not conducted. Based on these data, it was possible to identify and discuss weaknesses that must be overcome in order to strengthen the PRMSC. Conclusion: It is concluded that the evaluation instruments are important for the identification of points that need to be improved within the program, using the perception of the sample inserted in the program for directions that aim to promote improvements in the weaknesses found, thus enabling the strengthening and program development.

Keywords: Internship and Residency, Public Health, Program Evaluation. 


\section{INTRODUÇÃO}

O Sistema Único de Saúde (SUS) surgiu no Brasil como proposta de sistema universal de saúde, propondo às instituições formadoras utilizarem os serviços de saúde em seu contexto real como cenário de prática, diversificando e estruturando um modelo de formação capaz de contemplar as dimensões: técnica, científica, humana e política. Nesse aspecto, temos os Programas de Residência em Saúde como modalidade de ensino e formação, que agregam essas características, possuindo o trabalho como eixo condutor, e os serviços de saúde como cenário de prática (SOARES et al., 2018).

Os programas de residências multiprofissionais e em área profissional da saúde, foram criadas após a publicação da Lei n 11.129 de 2005. Os programas são norteadas pelos princípios e diretrizes do SUS, levando em consideração as necessidades e realidades locais e regionais, abrangendo diversas profissões da área da saúde, como: Biomedicina, Ciências Biológicas, Educação Física, Enfermagem, Farmácia, Fisioterapia, Fonoaudiologia, Medicina Veterinária, Nutrição, Odontologia, Psicologia, Serviço Social e Terapia Ocupacional segundo a resolução CNS no 287 (BRASIL, 1998).

A Comissão Nacional de Residência Multiprofissional em Saúde - CNRMS, criada através da Portaria Interministerial ㄲ⒈077, de 12 de novembro de 2009, é coordenada, em conjunto, pelo Ministério da Saúde e pelo Ministério da Educação e tem como funções principais: avaliar e creditar os programas de Residência Multiprofissional em Saúde e Residência em Área Profissional da Saúde conforme os princípios e diretrizes do SUS e que atendam às necessidades sócio epidemiológicas da população brasileira (BRASIL, 2019).

Além disso, habilitar os programas de Residência Multiprofissional em Saúde e Residência em Área Profissional da Saúde e também as instituições credenciadas para o oferecer; registrar certificados de Programas de Residência Multiprofissional 
em Saúde e Residência em Área Profissional da Saúde, de legitimidade nacional, com especificação de categoria e ênfase do programa (BRASIL, 2019).

As residências multiprofissionais em saúde devem ser afirmadas como importantes instrumentos na formação de profissionais verdadeiramente capacitados para o sistema público de saúde nacional. Durante pouco mais de uma década foram constituídas diversas propostas, promulgando avanços e retrocessos, disputas e conquistas inerentes da implementação do SUS e da vigência de projetos de saúde contrapostos.

A proposta da residência como uma modalidade de formação permite vivenciar e problematizar realidades estagnadas, propondo a criação e desenvolvimento de ações a partir de conhecimentos adquiridos por meio de experiência vivenciada, bem como processos reflexivos sobre a atuação profissional que surgem a partir dessas experiências. A partir desses processos reflexivos e críticos sobre a vivência, surge a possibilidade de mudanças. A renovação dos trabalhos em saúde se dá pela união entre os novos saberes e as experiências adquiridas, abrindo assim novos caminhos que viabilizam as melhorias (MIOTO et al., 2015).

Os Programas de Residência Multiprofissional em Saúde (PRMS) são mecanismos de cooperação intersetorial, que visam à inserção de jovens profissionais da saúde qualificados para exercer suas profissões no mercado de trabalho, particularmente preparados para trabalhar e contribuir para o desenvolvimento e construção do SUS (ANDRADE et al., 2018).

Programa de Residência em Saúde Coletiva (PRSC) com foco em gestão e planejamento em saúde, em particular, permite projetar a habitual lógica reproduzida no dia a dia dos serviços de saúde. Um dos valores da RMSC que tem sido reforçado em atividades cotidianas é desenvolver e melhorar as práticas coletivas, que promovam o enfrentamento às ações fragmentadas advindas das formações iniciais, acrescenta-se também o compromisso com os princípios e diretrizes do SUS, principalmente no que se refere à integralidade (SOARES et al., 2018).

Levando em consideração a importância da Residência Multiprofissional em Saúde Coletiva (RMSC) na região em que está inserida, esta pesquisa pode gerar conhecimentos sobre a temática da Residência Multiprofissional, oferecendo, assim, 
subsídios para o meio acadêmico, potencializando a criação de novos programas de residência e agregando valor aos profissionais envolvidos no programa (gestores, tutores e residentes), nos permitindo compreender o programa implantado no sertão da Paraíba e utilizar um instrumento qualificador para a sua avaliação, visando contribuir para o monitoramento, desenvolvimento e melhoria do programa.

Nestas perspectivas, o objetivo do estudo foi avaliar a atuação, atividades, a interação entre os profissionais e a estrutura organizacional de um PRM situado no alto sertão da Paraíba.

\section{METODOLOGIA}

O estudo transversal desenvolvido tratou-se de um estudo de quantitativo, descritivo que avaliou a percepção dos coordenadores, tutores, residentes e preceptores sobre o Programa de Residência Multiprofissional em Saúde Coletiva do alto sertão da Paraíba.

Os sujeitos da pesquisa foram profissionais inseridos no PRMSC vinculado a Faculdade Santa Maria de Cajazeiras, situada no alto sertão da Paraíba, sendo a mostra composta por: 2 coordenadores, 3 preceptores, 21 residentes e 7 tutores, que receberam o instrumento avaliativo de forma online. Dessa amostra de 33 pessoas, obtivemos 29 respostas ao questionário. A pesquisa foi aprovada pelo Comitê de Ética e Pesquisa da Faculdade Santa Maria, número do parecer 3.833.145, seguindo os preceitos éticos determinados na Resolução no 510/16.

Foram utilizados como critério de inclusão os profissionais inseridos no Programa de Residência Multiprofissional em Saúde Coletiva incluindo gestores, tutores e residentes (TCLE). Os profissionais que preencheram o questionário incompleto, foram excluídos do estudo.

O questionário utilizado para coleta de dados foi elaborado a partir do estudo de Bezerra et al. (2016), que fizeram um instrumento avaliador onde a grande maioria dos indicadores era compatível com o modelo lógico proposto e atendia aos objetivos da avaliação. Os resultados desse estudo foram obtidos através de um 
instrumento de coleta de dados composto de 27 questões objetivas, divididas em três dimensões capazes de avaliar o programa de residência englobando três diferentes áreas, a área de gestão do programa, a de estrutura organizacional, e a área de processo pedagógico.

A análise estatística ocorreu através do programa software Statistical Package for Social Science (Versão 22.0, IBM SPPS Inc., Armonk, NY, USA). Empregou-se a estatística descritiva, por meio de frequência absoluta e relativa para caracterização da amostra e distribuição das variáveis do estudo.

\section{RESULTADOS E DISCUSSÃO}

A amostra contava com 33 participantes, porém só 29 participaram da pesquisa respondendo ao questionário aplicado.

A Dimensão I contava com 15 perguntas sobre a gestão do Programa De Residência Multiprofissional em Saúde Coletiva. O objetivo desta dimensão é avaliar o programa desde os parâmetros de sua implantação até o desenvolvimento e aplicação os seus instrumentos de gestão.

Dentro da Dimensão I, no primeiro quesito (Gráfico 1), percebe-se o resultado perceptivo da amostra sobre o grau de envolvimento entre tutores, preceptores e IES na implantação do PRMSC, onde 50\% consideraram que houve um envolvimento parcial na implantação. Diante dos dados obtidos, podemos notar que a grande maioria dos envolvidos no programa não teve participação ativa na implantação do PRMSC.

Mattos (2016) constatou em sua pesquisa que muitas vezes a implantação da Residência Multiprofissional em Saúde é realizada de forma impositiva, não havendo um tempo suficiente para a uma adequada estruturação. Assim, o envolvimento dos tutores e preceptores ocorre sem o devido protagonismo e formação pedagógica requerida. Isto poderá causar certa fragilidade no desenvolver das práticas educativas pelas dificuldades em desenvolver a educação permanente, que é o eixo norteador e pré-requisito para implantação da RMS. 


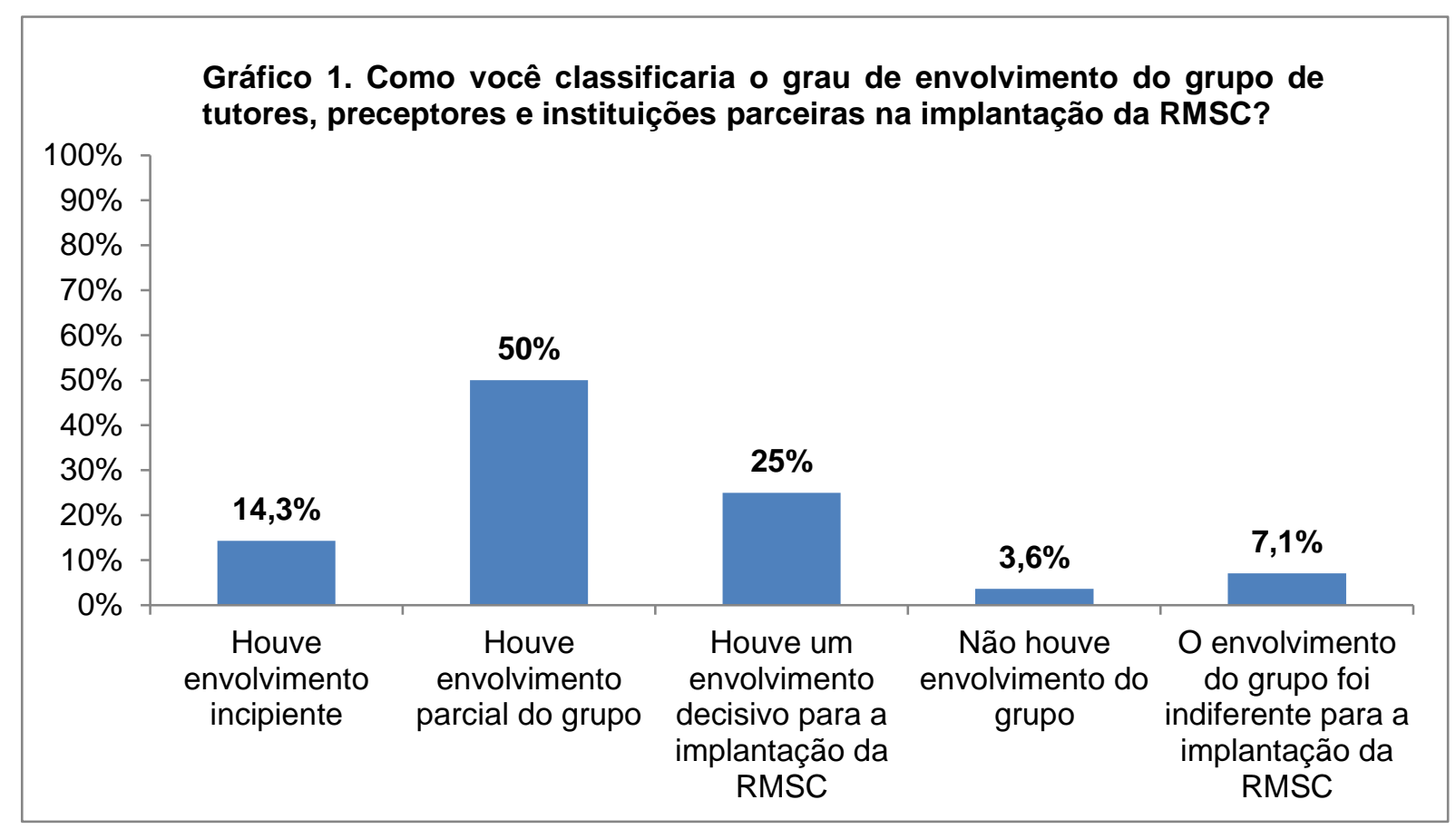

Fonte: Do Autor.

No quesito vínculo entre a IES com a RMSC, a totalidade dos participantes respondeu que esse vínculo se faz presente, quando levado em consideração o envolvimento da IES para o desenvolvimento da RMSC (Gráfico 2), 46,4\% consideraram que houve um envolvimento parcial da IES para o desenvolvimento da RMSC. 


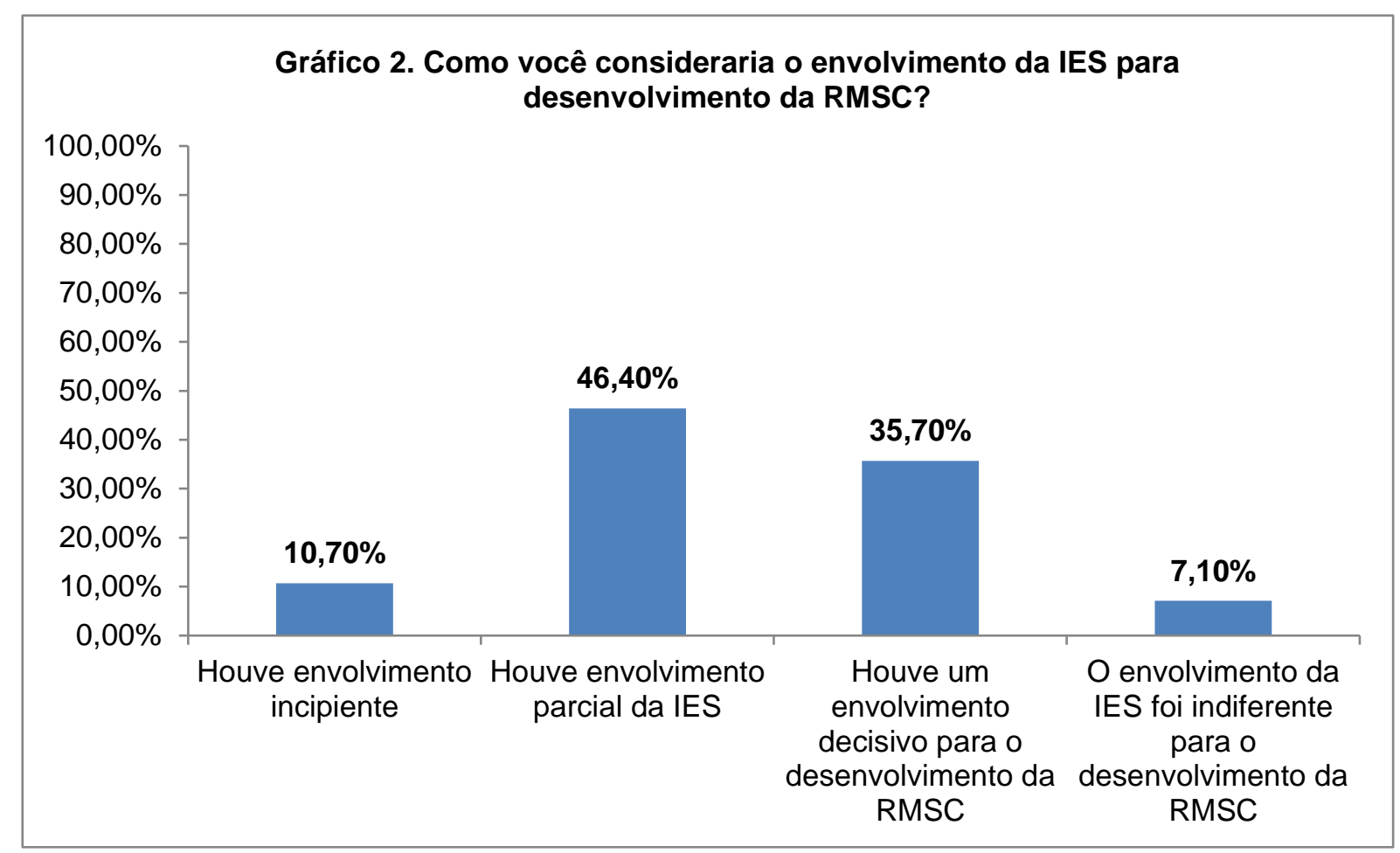

Fonte: Do Autor.

$\mathrm{Na}$ questão que aborda o quesito integração entre o PRMSC e os outros programas de residência regionais (Gráfico 3), 39,3 \% consideraram existir pouca integração, 35,7\% consideraram não existir integração.

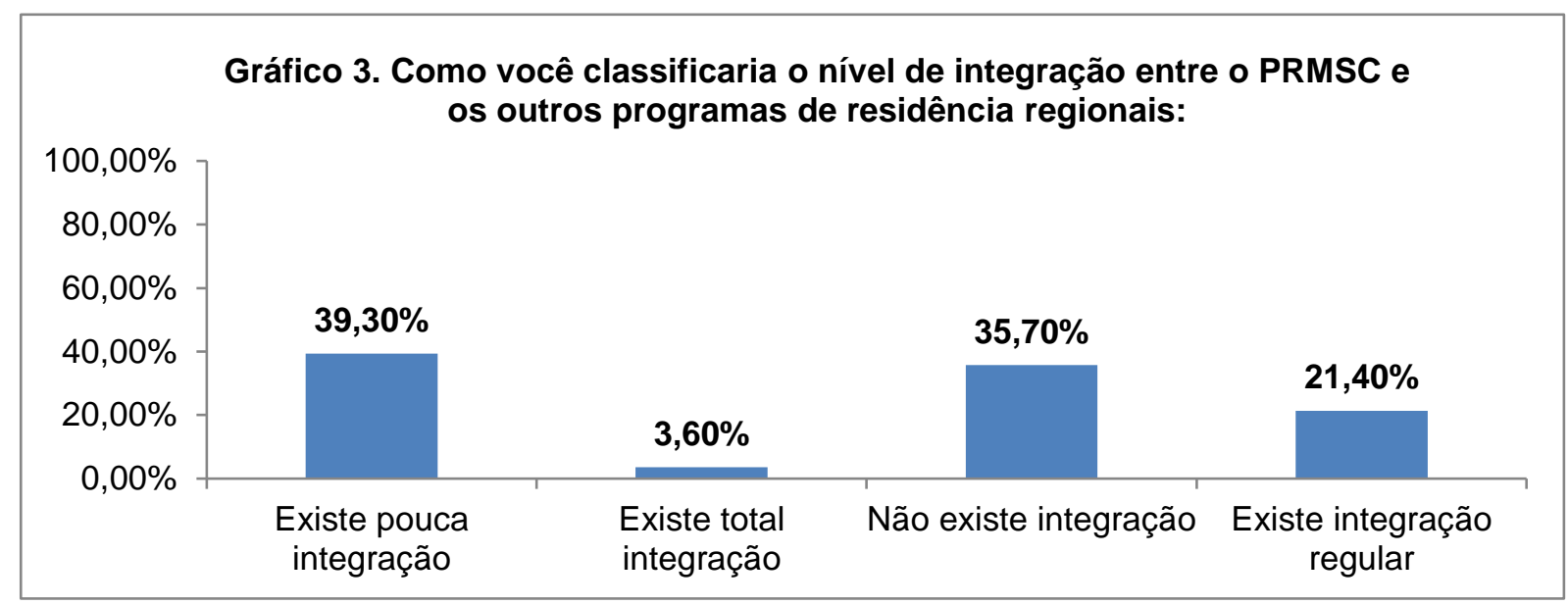

Fonte: Do Autor.

Torres et al. (2019) em sua pesquisa ressaltaram a importância de uma maior interlocução e vivência entre diferentes programas de Residência em Saúde, que se 
configura como uma forma de possibilitar a busca pela integralidade em saúde. Participar de um processo formativo que se baseia na construção articulada de diferentes áreas e profissões da saúde, buscando construir um saber comum, onde se agreguem as contribuições dos diferentes núcleos profissionais, efetiva, de fato, uma prática renovadora na saúde.

Em relação à realização de avaliação sistemática do PRMSC, 28,6\% consideraram que essas avaliações não são previstas e nem realizadas (Gráfico 4).

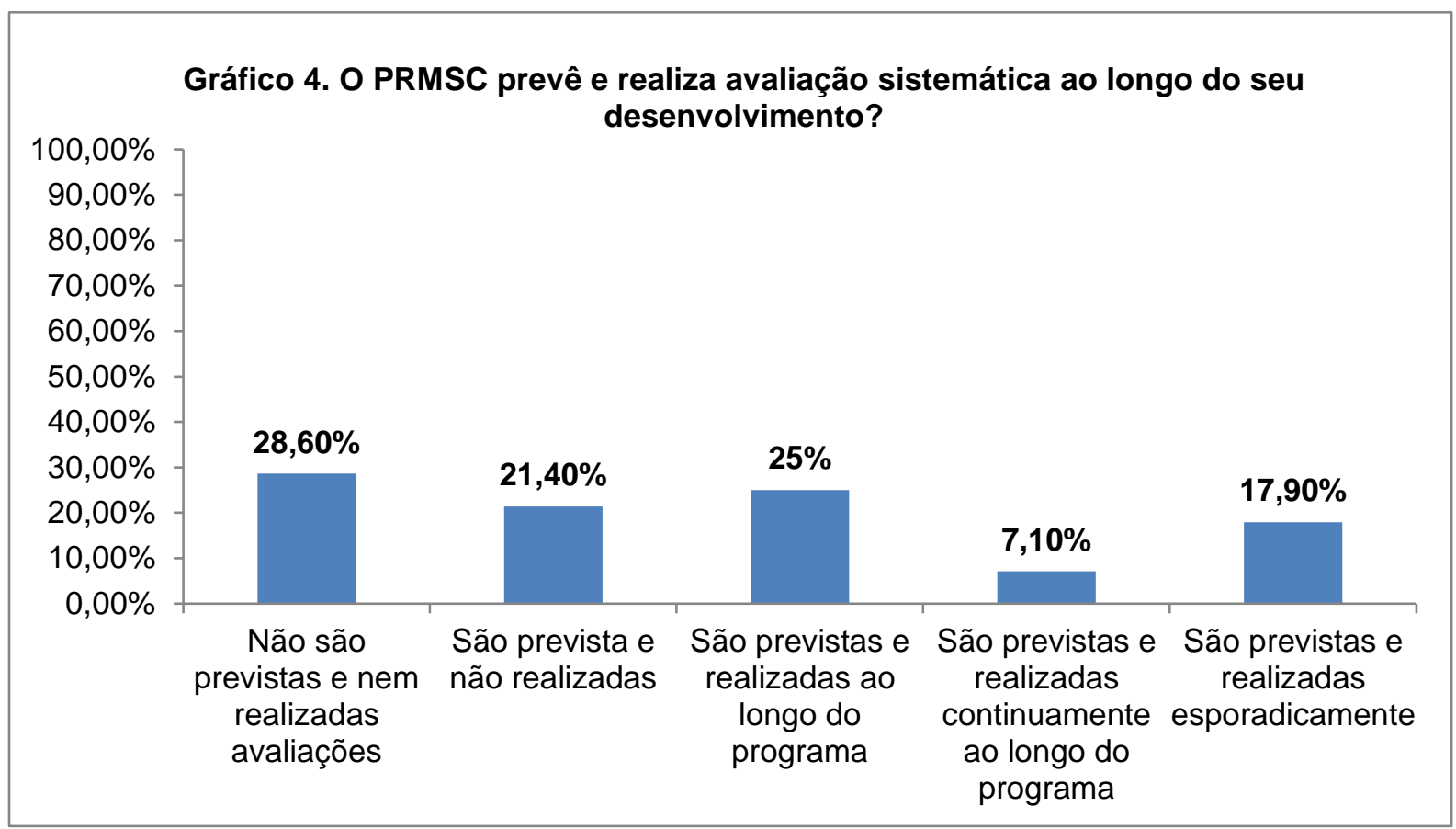

Fonte: Do Autor.

$\mathrm{Na}$ vertente da participação dos residentes, tutores e preceptores nas avaliações sistemáticas do PRMSC, 42,9\% consideraram que existe pouca participação (Gráfico 5). 


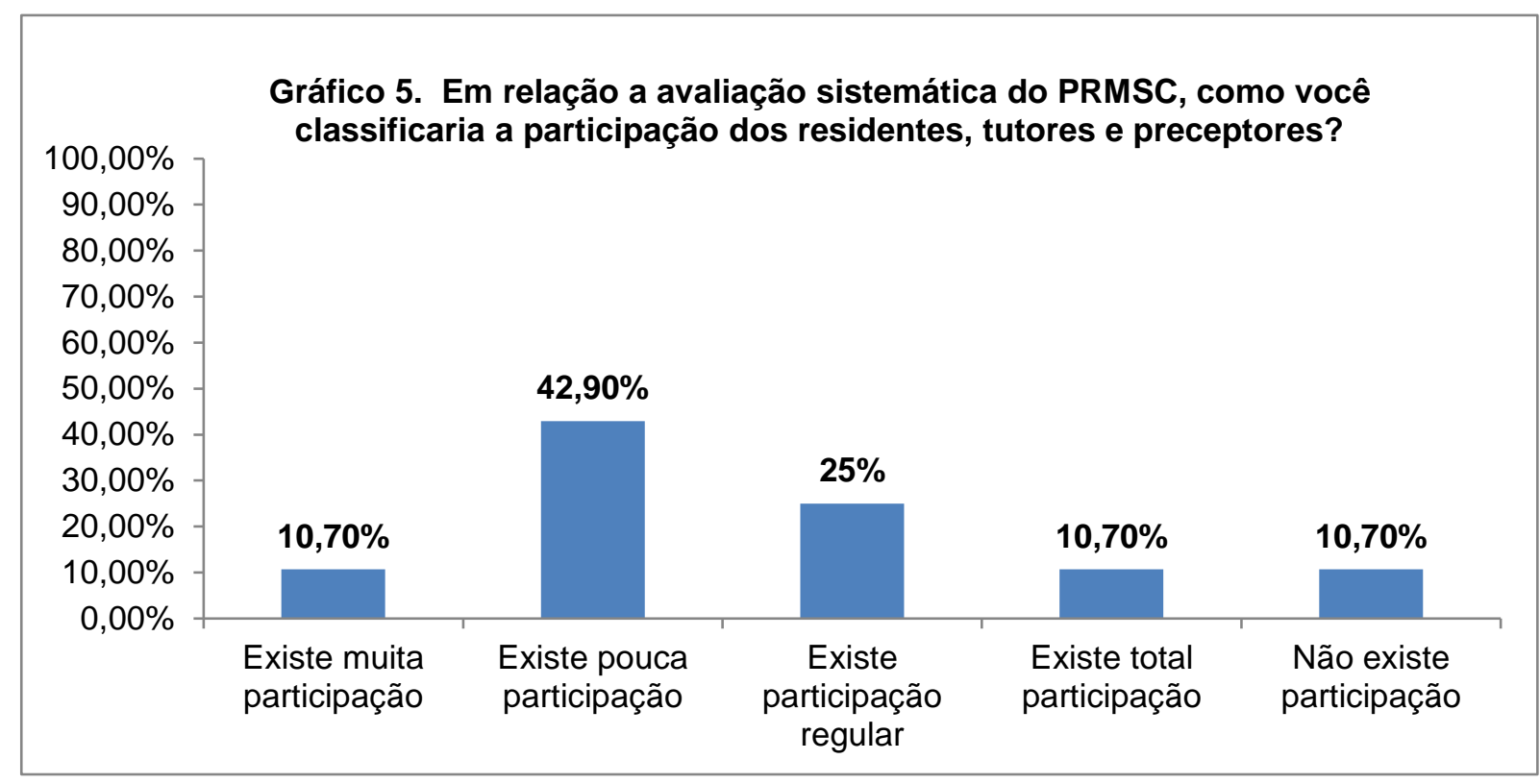

Fonte: Do Autor.

Segundo Lima e Passos (2019), é de extrema importância a realização de avaliações sistemáticas dos programas de residência em saúde, pois esse modelo de formação exige constantes adequações e transformações práticas, sendo necessária a reformulação do processo de formação e de novas invenções. Também é algo característico da educação permanente, assim, a partir da identificação e análise de um problema ou vulnerabilidade, é possível a busca pela melhoria, para resolver aquele ponto e construir junto com a equipe local a reformulação de algumas práticas.

Em relação à garantia de acesso aos materiais didáticos pelos preceptores, tutores e residentes, 35,7\% consideraram que nenhum material é garantido, enquanto no quesito de escolaridade efetiva, que inclui a emissão de certificados, de declaração, controle de frequência, entre outros, $46,4 \%$ consideraram esse tipo de serviço do PRMSC bom.

Sobre o regimento interno do PRMSC, no quesito de participação dos residentes, tutores e preceptores na elaboração e aprovação desse regimento, $32,1 \%$ consideraram existir pouca participação e $25 \%$ consideraram não existir participação (Gráfico 6). 


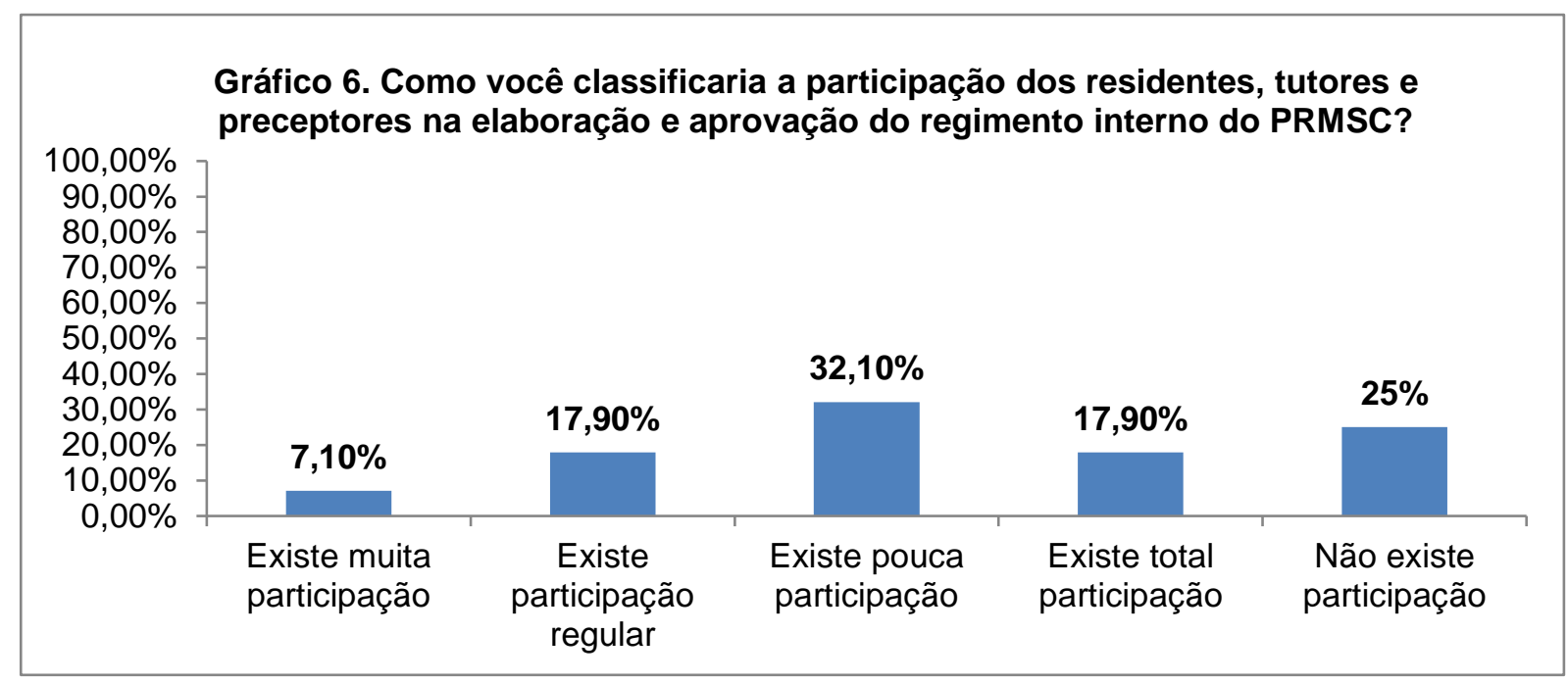

Fonte: Do Autor.

Levando em conta a utilização do regimento interno como instrumento norteador nas ações do programa, 42,9\% consideraram que a utilização acontece apenas quando demandada (Gráfico 7).

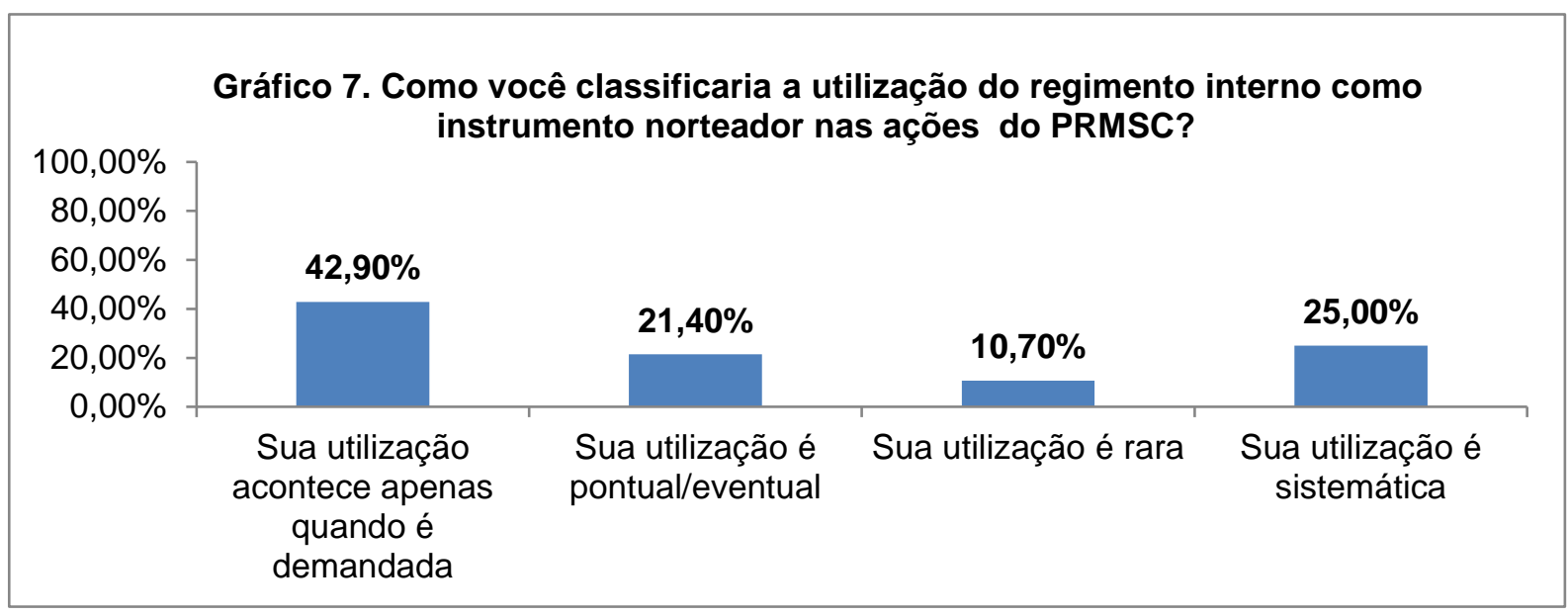

Fonte: Do Autor.

Considerando que todos os programas de residências devem ter seu regimento interno, que tem por finalidade orientar e disciplinar o funcionamento do programa, Silva e Natal (2019) afirmam que ainda que este não seja formulado e apresentado formalmente para aos envolvidos no programa, este deve ser usado como instrumento norteador durante o processo, pois contém as informações necessárias sobre a organização e proposta de condução do programa, sendo 
assim a sua não divulgação é um ponto negativo para a adoção do papel do preceptor e tutor. Vale ressaltar que o regimento interno pode sofrer alterações a fim de promover o fortalecimento do programa, desde que essas alterações sejam aprovadas pelos colegiados responsáveis.

Quanto à experiência e qualificação dos coordenadores, tutores e preceptores, $32,1 \%$ consideraram que entre 40 e $60 \%$ seriam experientes e qualificados para tais atividades.

No quesito de distribuição de vagas por categoria profissional, o programa pode usar critérios predefinidos como: necessidade locorregional, demanda de candidatos, perfil epidemiológico, entre outros, no PRMSC, 42,9\% consideraram que essa distribuição ocorre sem critérios predefinidos (Gráfico 8).

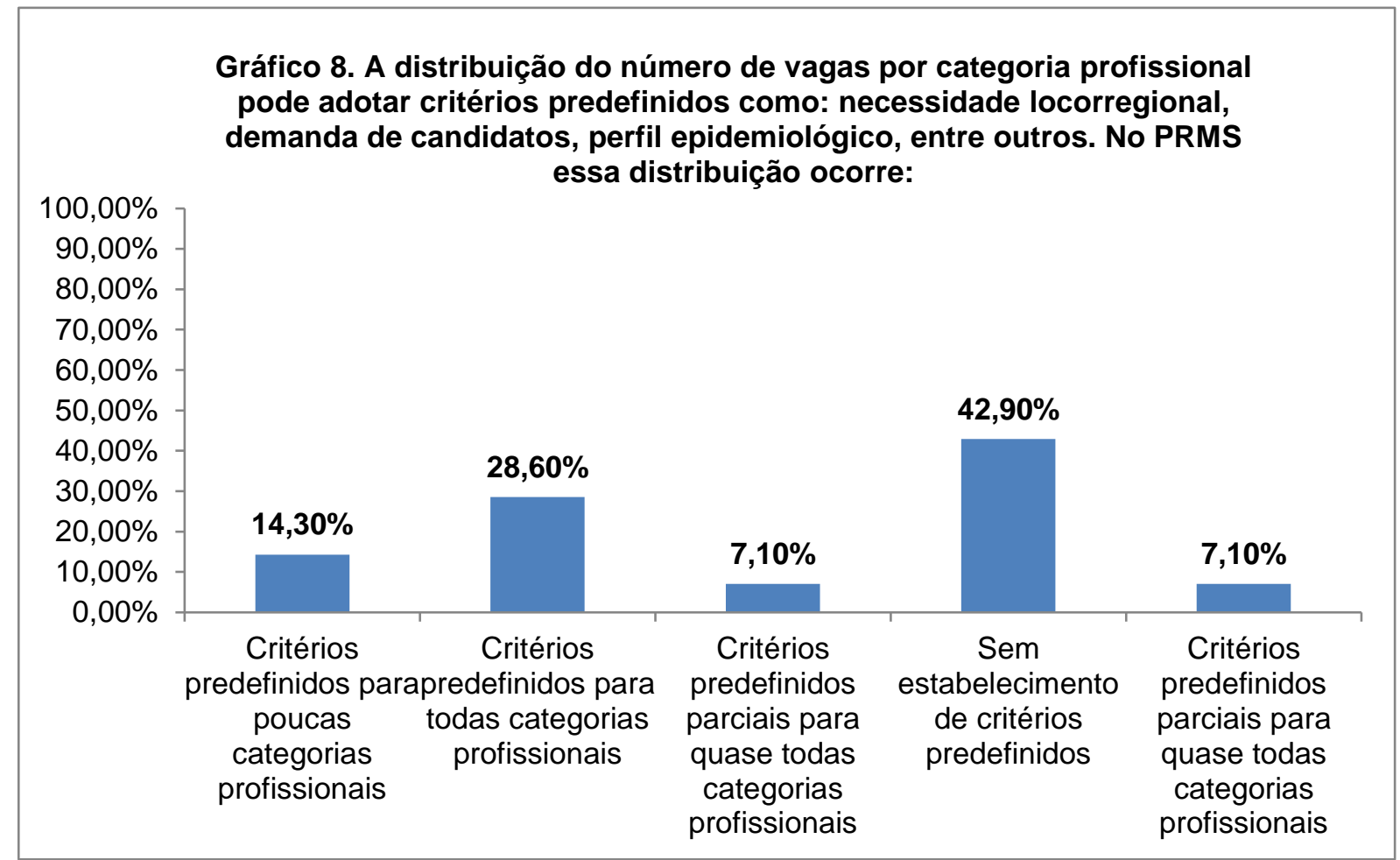

Fonte: Do Autor.

No Brasil existe um consenso que a má distribuição de serviços e profissionais de saúde vem dificultando a consolidação do sus, o estudo realizado por Sarmento et al. (2017) considera os Programas de Residência em Saúde como meio fixador de profissionais, assumindo assim um importante papel como regulador da oferta para áreas consideradas prioritárias para o SUS. 
Para isso, torna-se necessário a adoção de critérios definidos a partir de estudos locorregionais, utilizando a descentralização, a regionalização e a interiorização como eixos norteadores para a distribuição desses programas e consequentemente do número de profissionais necessários para cada região à que está vinculado.

Levando em conta a participação dos tutores, residentes e preceptores na Comissão de Residência Multiprofissional (COREMU), 28,6\% consideraram existir participação de todos e 28,6\% que existe participação regular (Gráfico 9).

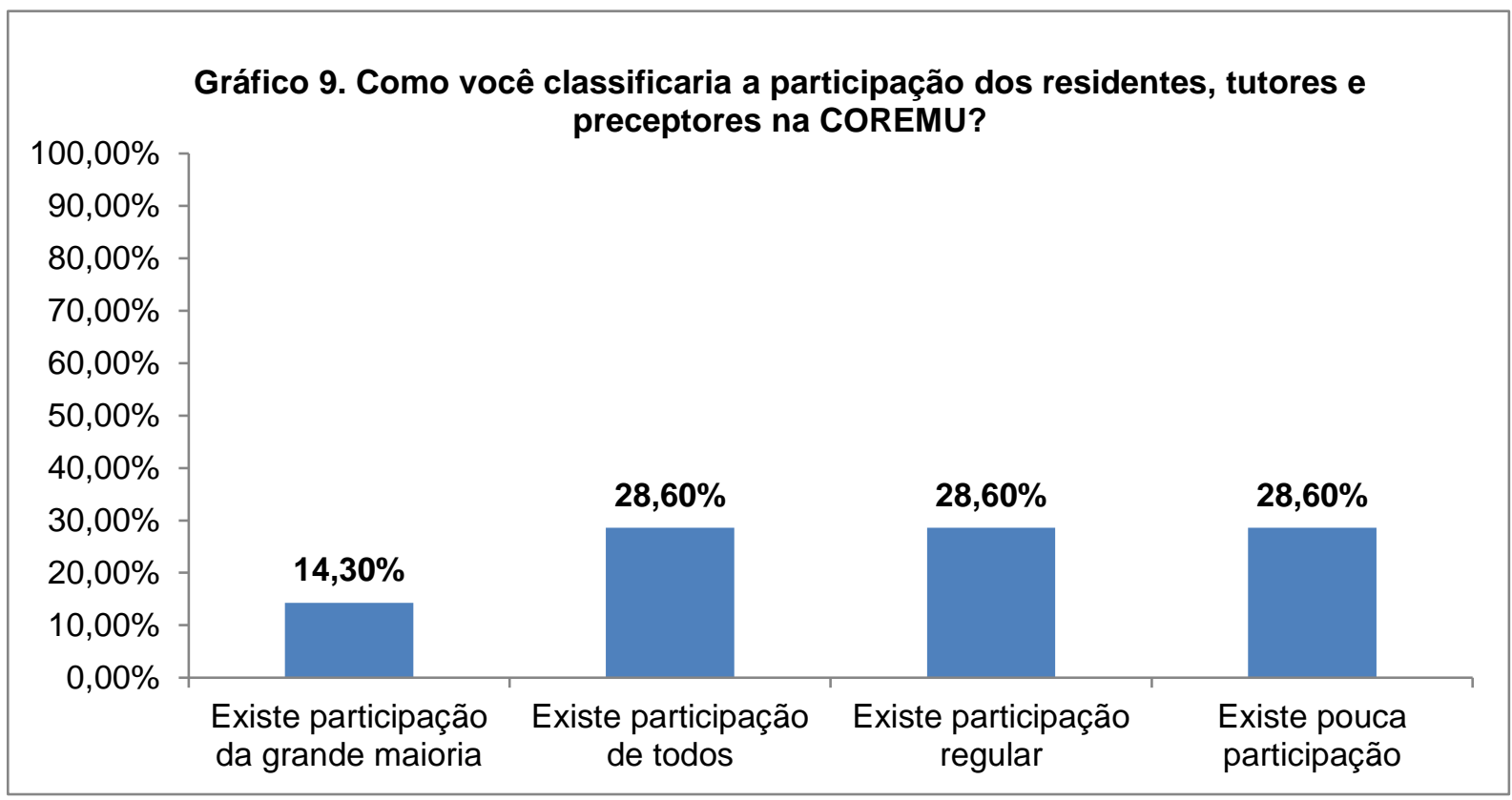

Fonte: Do Autor.

A COREMU é o órgão deve se responsabilizar pelas ações de um Núcleo Docente-Assistencial Estruturante (NDAE) composto por docentes, tutores, preceptores e profissionais da saúde residentes. Silva e Natal (2019) consideram as reuniões do NDAE e da COREMU instrumentos potencializadores contando com a representatividade da amostra, além do acesso livre às atas destas reuniões, sendo um espaço de tomada de decisões através de um planejamento válido e construído por todos.

A Dimensão II do instrumento mostra a percepção dos envolvidos no programa sobre a estrutura organizacional do PRMSC, quando perguntados sobre as salas de aulas disponíveis $82,2 \%$ consideraram entre ótimo e bom. Quanto à presença de sala de auditório disponíveis, 78,6\% consideraram entre ótimo e bom. 
Sobre o monitoramento de infraestruturas dos cenários de prática, 28,6\% consideraram não existir monitoramento (Gráfico 10).

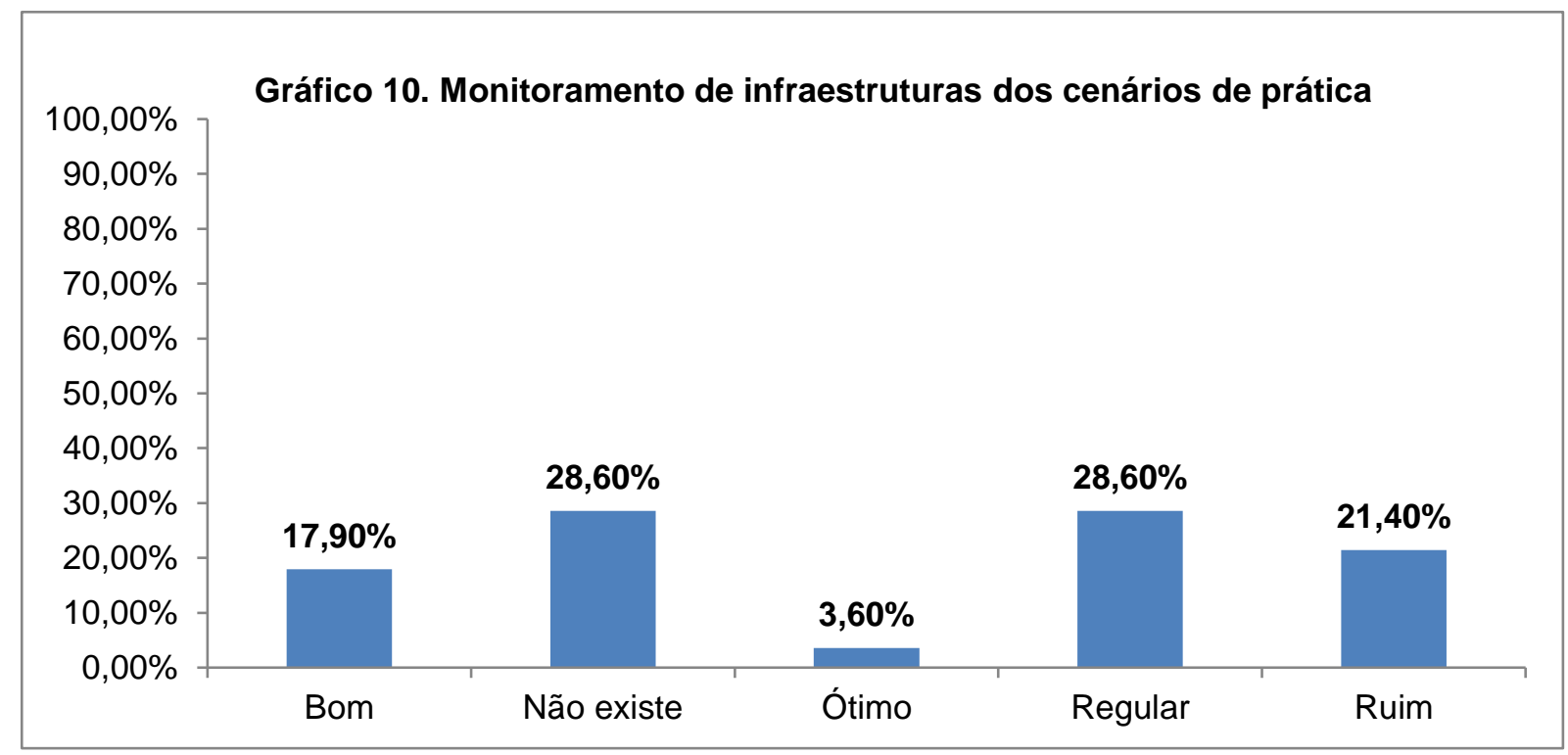

Fonte: Do Autor.

Diante dos dados obtidos, podemos verificar que existe uma necessidade de melhoria no monitoramento dos cenários de práticas, porém é importante frisar que dentro do Programa de Residência Multiprofissional em Saúde Coletiva onde os cenários de práticas são os serviços do SUS, a melhoria de infraestruturas torna-se algo que foge da governabilidade da coordenação do programa.

Devemos considerando os problemas estruturais enfrentados pelo SUS decorrentes de financiamento inadequado para as demandas encontradas, gestão não qualificada para gerir recursos existentes, sucateamento dos serviços de saúde, que necessitam de melhorias nas políticas de financiamentos e monitoramentos de gastos públicos para haver uma reversão desse quadro vivido pelo SUS.

A Dimensão III do instrumento avaliativo nos permite analisar a percepção dos autores sobre o Processo pedagógico desenvolvido na RMSC.

O primeiro quesito desta dimensão avalia a oferta da Educação Permanente para tutores e preceptores do PRMSC, no qual 50\% consideraram que a educação permanente é ofertada de forma pontual quando demanda pelo programa (Gráfico 11). 


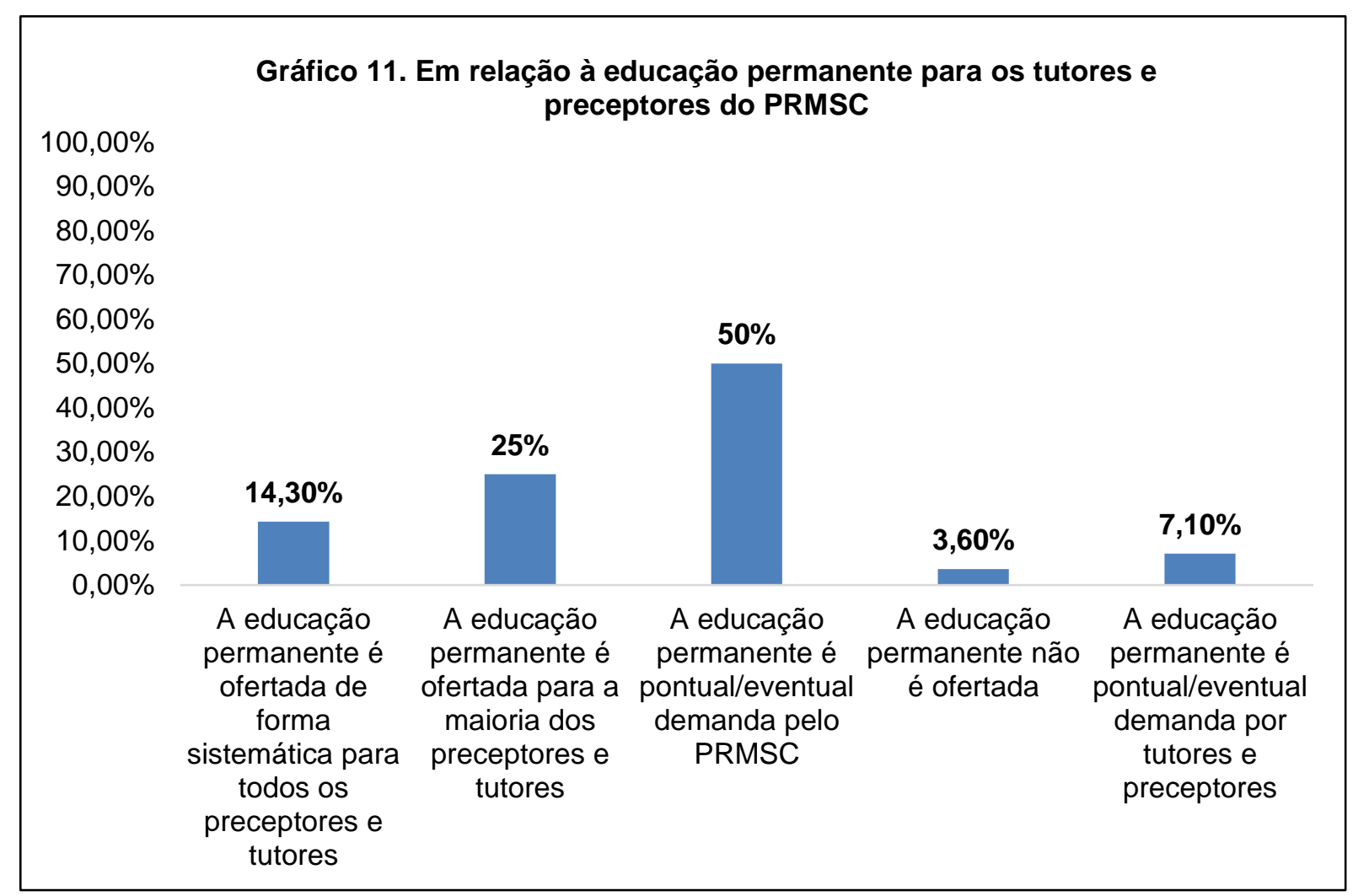

Fonte: Do Autor.

Considerando que os Programas de Residências em Saúde devem utilizar a educação permanente como principal eixo na formação dos profissionais, Silva et al. (2016) afirmam que os programas de residência atuam como disseminadores da educação permanente entre os profissionais de saúde, usuários, docentes e estudantes, porém destaca que a sua utilização, na maioria das vezes, ocorre de forma pontual, identificando assim que ainda há muitos desafios para que de fato ocorra a disseminação e consolidação da educação permanente como política para formação dos profissionais de saúde. Tal política permite que o desenvolvimento de profissionais de saúde ocorra de modo descentralizado, ascendente e transdisciplinar, englobando todos os locais e saberes a fim de proporcionar a democratização dos espaços de trabalho.

$\mathrm{Na}$ perspectiva de utilização do Projeto pedagógico como instrumento norteador das atividades desenvolvidas no PRMSC, 42,90\% consideraram que a articulação entre o projeto pedagógico e os conteúdos teóricos e práticos como regular (Gráfico 12). 


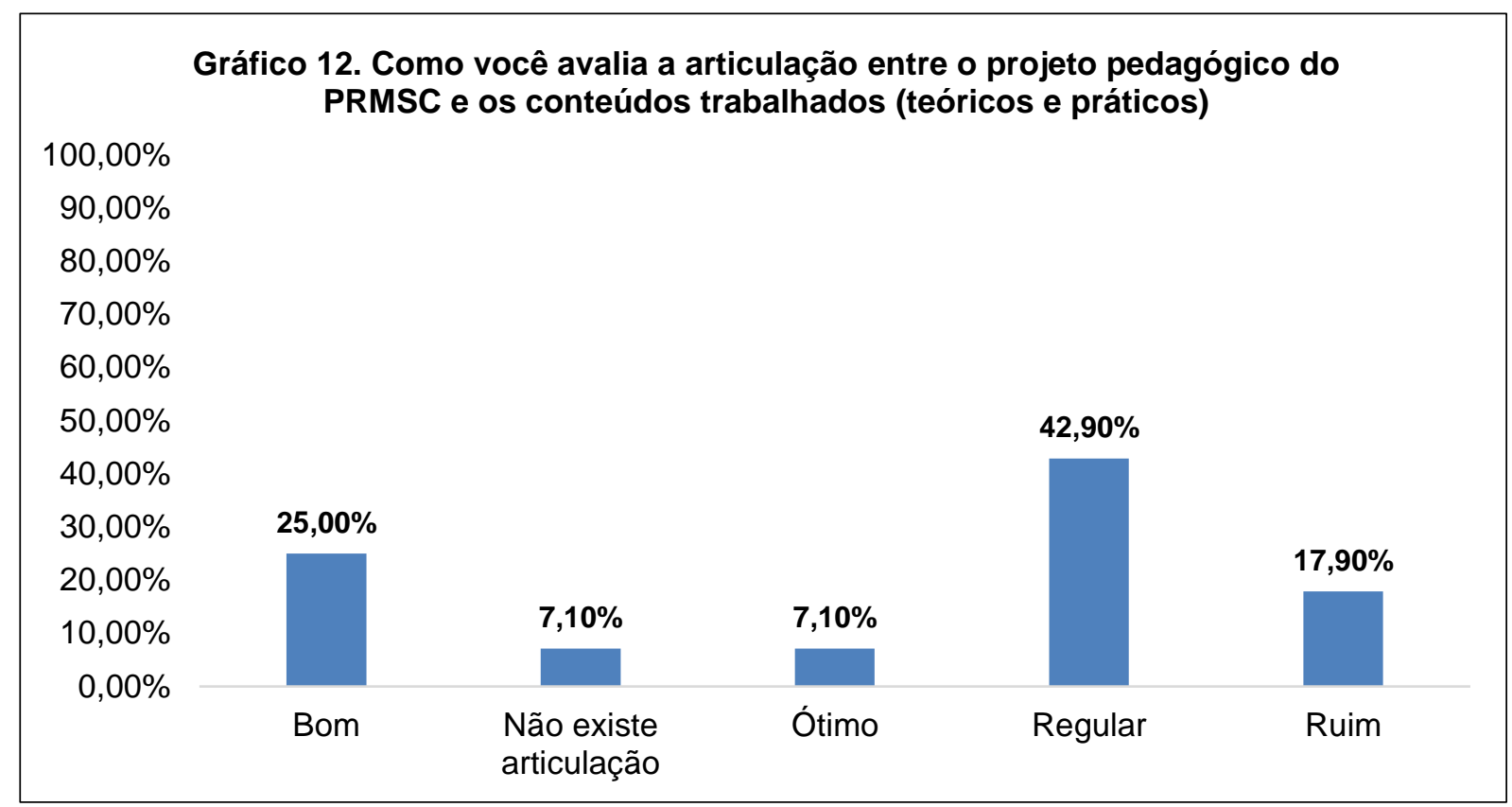

Fonte: Do Autor.

Os Projetos Pedagógicos são a expressão dos valores e princípios educacionais e políticos adotados pelos programas, frutos de uma produção coletiva, com o objetivo de nortear e conduzir o processo de formação, buscando superar desafios políticos e pedagógicos. Miranda et al. (2015) citam a prática profissional como eixo norteador do projeto pedagógico e das metodologias de ensino, e a flexibilidade do plano pedagógico que deve ser constantemente avaliado para que possa ser reconstruído e discutido de acordo com as devolutivas.

Sobre o processo de avaliação do PRMSC, 28,6\% dos profissionais envolvidos consideraram que as avaliações são previstas e realizadas ao longo do programa, entretanto $28,6 \%$ consideraram também que essas avaliações são previstas e não realizadas (Gráfico 13). 


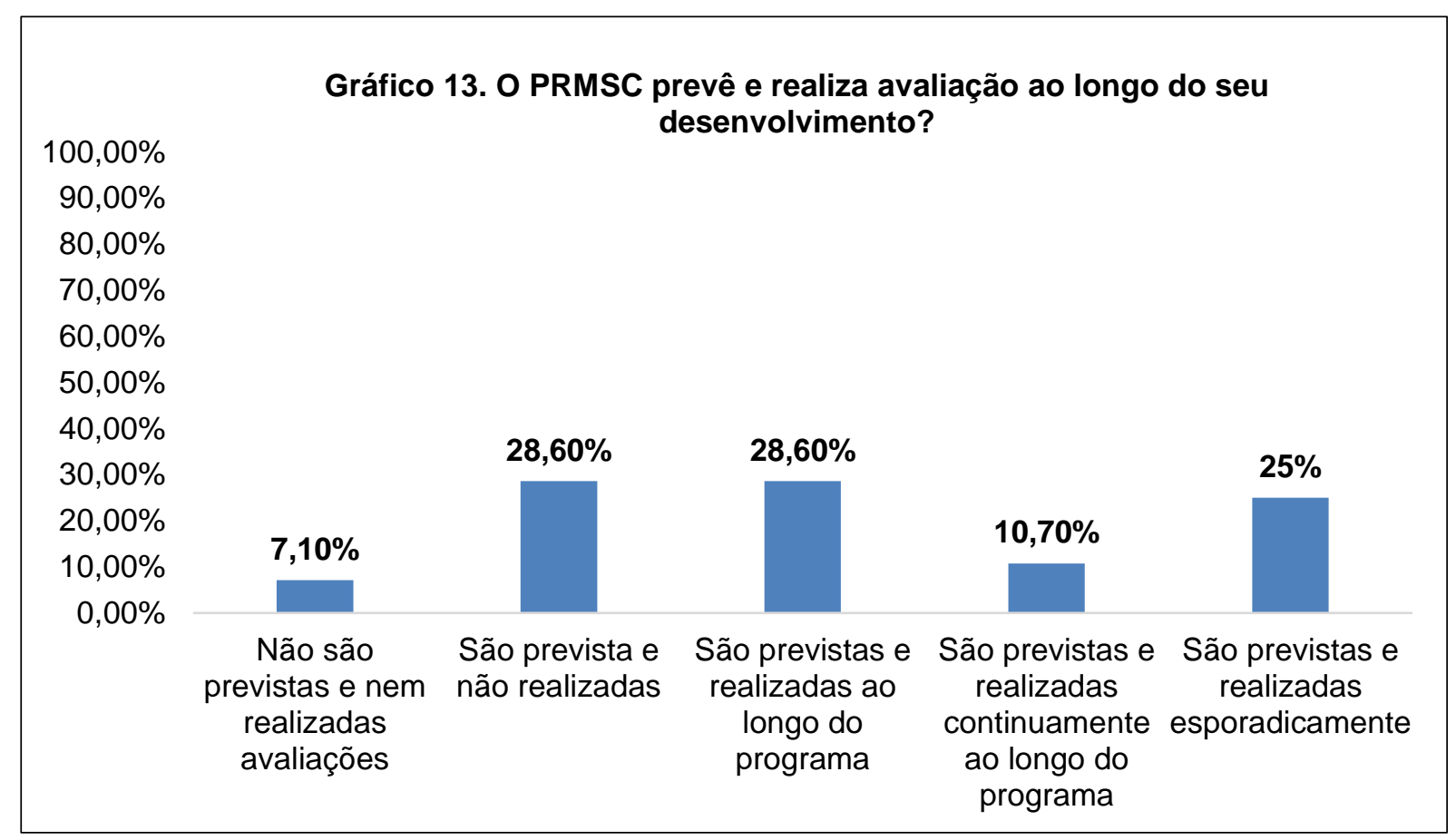

Fonte: Do Autor.

Diante dos dados, é importante ressaltar que a avaliação do PRMSC está prevista dentro do regimento interno e do projeto pedagógico, e deve ser feita ao longo do processo da RMSC. Os instrumentos avaliativos são de extrema relevância para a identificação das fragilidades encontradas no decorrer do programa, sendo um meio expositor, no qual os profissionais envolvidos no processo podem qualificar o programa a partir das suas práticas vivenciadas. Esse instrumento de avaliação desse servir como norteador para propostas de mudanças que tragam melhorias e ajudem no desenvolvimento do PRMSC.

O desenho de estudo é um fator limitador do presente trabalho, além da realização da pesquisa com um único programa de residência. Dessa forma, estudos futuros com desenhos mais elaborados como estudos longitudinais e abranger outros programas poderão apresentar melhores resultados. 


\section{CONCLUSÃO}

O estudo realizado por meio da análise dos dados obtidos pelo instrumento avaliativo, mostra que o PRMSC possui algumas fragilidades que podem ser contornadas no decorrer do seu desenvolvimento.

Levando em consideração as três dimensões analisadas, vemos através da percepção da amostra inserida no programa, a necessidade da ampliação do diálogo entre os residentes, preceptores, tutores e coordenadores, para direcionamentos que visem promover melhorias nos pontos de fragilidades encontrados, possibilitando assim o fortalecimento e desenvolvimento do programa.

\section{REFERÊNCIAS BIBLIOGRÁFICAS}

ANDRADE, L.; ALVES, L.; CÂMARA, I.; DANTAS, M.; ALMEIDA, L. Da graduação à residência em saúde: refletindo sobre o processo de integração ensino-serviço na rede. Divulgação em Saúde para Debate, Rio de Janeiro, v.1, n. 58, p. 5-8, 2018.

BEZERRA, T.C.A.; FALCÃO, M.L.P.; GOES, P.S.A.; FELISBERTO, E. Avaliação De Programas De Formação Profissional Em Saúde: Construção E Validação De Indicadores. Trabalho, Educação e Saúde, Rio de Janeiro, v.14, n. 2, p. 445-472, 2016.

BRASIL. Conselho Nacional de Saúde. Resolução no 287, 08 de outubro de 1998. Diário Oficial da União (D.O.U), Brasília, 1998.

BRASIL. Ministério da Educação, Ministério da Saúde (BR). Portaria Interministerial $\mathbf{n} \mathbf{0} \mathbf{4 5}$, de 12 de janeiro de 2007. Dispõe sobre a Residência Multiprofissional em Saúde e a Residência em Área Profissional da Saúde e institui a Comissão Nacional de Residência Multiprofissional em Saúde. Diário Oficial da União [da] República Federativa do Brasil, 17 de janeiro de 2007.

BRASIL. Ministério da Educação. Residência multiprofissional. 2019. Disponível em: $<$ http://portal.mec.gov.br/residencias-em-saude/residencia-multiprofissional> acesso em: 01/10/2019.

GERHARDT, T. E.; SILVEIRA, D. T. Métodos de pesquisa; coordenado pela Universidade Aberta do Brasil - UAB/UFRGS e pelo Curso de Graduação Tecnológica - Planejamento e Gestão para o Desenvolvimento Rural da SEAD/UFRGS. - Porto Alegre: Editora da UFRGS, 2009

LIMA, I. C. B.; PASSOS, I. C. F., Residências integradas em saúde mental: para além do tecnicismo. Trabalho, educação e saúde, Rio de Janeiro, v. 17, n. 2, e0020940, 2019.

MARTINS, G. D. M.; CAREGNATO, R. C. A.; BARROSO, L. M.; RIBAS, D. C. P.; Implementação de residência multiprofissional em saúde de uma universidade federal: trajetória histórica. Rev. Gaúcha de Enfermagem, v. 37, n. 3, e57046, 2016. 
MIOTO, R. C. T.; ALVES, F. L.; CAETANO, P. S. As residências multiprofissionais em saúde: a experiência da Universidade Federal de Santa Catarina. Serviço Social e Saúde, v.11, n.2, p.185-208, 2015.

MIRANDA, N. M.V, LEONELLO, V. M., OLIVEIRA, M.A.C. Residências multiprofissionais em saúde: análise documental de projetos político-pedagógicos. Revista Brasileira de Enfermagem, v.68, n.4, p.586-93, 2015.

SARMENTO, L. F.; FRANÇA, T.; MEDEIROS, K. R.; SANTOS, M. R.; NEY, M. S. A distribuição regional da oferta de formação na modalidade Residência Multiprofissional em Saúde. Saúde Debate, v.41, n.113, p.415-424, 2017.

SILVA, L.S.; NATAL, S. Residência multiprofissional em saúde: análise da implantação de dois programas pela Universidade Federal De Santa Catarina. Brasil. Trabalho, Educação e Saúde, v.17, n.3, e0022050, 2019.

SOARES, C. L. M.; VILASBÔAS, A. L. Q.; NUNES, C. A.; SANTOS, L. Residência em Saúde Coletiva com concentração em planejamento e gestão em saúde: a experiência do Instituto de Saúde Coletiva da Universidade Federal da Bahia. Divulgação em Saúde para Debate, n. 58, p. 5-8, jul 2018.

SOUZA, L.E. Qual a diferença entre saúde coletiva e saúde pública? Criada em 21/07/15 08:41. Atualizada em 21/07/15 12:05. Disponível em: < https://www.ufg.br/n/82100-saiba-adiferenca-entre-saude-coletiva-e-saude-publica> acesso em: 01/10/2019.

TORRES, R. B. S., et al. Estado da arte das residências integradas, multiprofissionais e em área profissional da Saúde. Interface, v.23, e170691, 2019. 